\title{
Heats of Transport from the Diffusion Thermoeffect in Binary Liquid Mixtures of Toluene, Chlorobenzene, and Bromobenzene
}

\author{
Richard L. Rowley
}

rowley@byu.edu

M. D. Hall

Follow this and additional works at: https://scholarsarchive.byu.edu/facpub

Part of the Chemical Engineering Commons

\section{Original Publication Citation}

R.L. Rowley and M.D. Hall, "Heats of Transport from the Diffusion Thermoeffect in Binary Liquid Mixtures of Toluene, Chlorobenzene, and Bromobenzene" J. Chem. Phys. 85, 355 (1986)

\section{BYU ScholarsArchive Citation}

Rowley, Richard L. and Hall, M. D., "Heats of Transport from the Diffusion Thermoeffect in Binary Liquid Mixtures of Toluene, Chlorobenzene, and Bromobenzene" (1986). Faculty Publications. 751.

https://scholarsarchive.byu.edu/facpub/751 


\title{
Heats of transport from the diffusion thermoeffect in binary liquid mixtures of toluene, chlorobenzene, and bromobenzene
}

\author{
R. L. Rowley and M. D. Halla),b) \\ Department of Chemical Engineering, Brigham Young University, Provo, Utah 84602
}

(Received 7 May 1986; accepted 10 June 1986)

\begin{abstract}
Measurements of heats of transport in binary liquid mixtures of toluene, chlorobenzene, and bromobenzene utilizing the diffusion thermoeffect, or Dufour effect, are reported at 298 and $308 \mathrm{~K}$. The corresponding heat-mass Onsager coefficients are also reported, from which thermal diffusion ratios have been calculated based on Onsager reciprocity. The resultant binary thermal diffusion ratios have been correlated as a function of composition in an attempt to study the relationship between values in binary and ternary mixtures. It was found that the single independent binary values can be used to estimate the two independent thermal diffusion ratios previously reported in toluene + chlorobenzene + bromobenzene ternary mixtures.
\end{abstract}

\section{INTRODUCTION}

Not only will a temperature gradient give rise to a heat flux in fluid mixtures, but also so will composition gradients (more appropriately, isothermal chemical potential gradients). This latter effect, known as the diffusion thermoeffect or the Dufour effect, is characterized by a transport property called the heat of transport. The heat of transport of component $i, Q_{i}^{*}$, relates the magnitude of the produced heat flux to the diffusional flux of component $i$ under isothermal conditions. Generally the diffusion thermoeffect is considered to be small. Previous studies ${ }^{1,2}$ have shown that while it is true that measured temperature gradients induced in initially isothermal liquid mixtures are small due to opposition by thermal conduction, the heat of transport itself is generally rather large, often on the order of several $\mathrm{kJ} / \mathrm{mol}$. As heats of transport contain significant information concerning the actual energy-diffusional relationship of molecular interactions, they are of interest in the development of molecular theory and generalized transport models.

Recently, Platt et al. ${ }^{2}$ have reported heats of transport in ternary liquid mixtures for the system toluene + chlorobenzene + bromobenze. In ternary mixtures, there are two independent heats of transport, ${ }^{3}$ or equivalently, two independent Onsager coefficients. Platt et al. ${ }^{4}$ reported Onsager coefficients obtained from their experimentally determined heats of transport, and then using Onsager reciprocity calculated the two independent thermal diffusion ratios $K_{T}$ as a function of composition in the ternary mixture. However, in binary systems there is only one independent heat of transport or corresponding heat-mass Onsager coefficient. This leads to the natural questions: what is the compositional behavior of the two independent Onsager coefficients in the binary limit and what is the relationship, if any, of the ternary heats of transport to the binary? The natural extension of the second question is, of course, can ternary heats of transport be predicted from binary measurements. In this

\footnotetext{
a) Present address: Western Geophysical, Houston, TX.

b) Experimental and thesis work performed at Department of Chemical Engineering, Rice University, Houston, TX.
}

work we report binary diffusion thermoeffect measurements made on binary mixtures consisting of toluene, chlorobenzene, and bromobenzene. From the results, binary heatmass Onsager coefficients and, based on Onsager reciprocity, thermal diffusion ratios are computed. The composition dependence of the binary values are then used in an attempt to correlate the ternary values reported by Platt $e t a l{ }^{2}$

\section{EXPERIMENTAL}

The diffusion thermoeffect technique for measurement of heats of transport was utilized in this study. As before, ${ }^{2}$

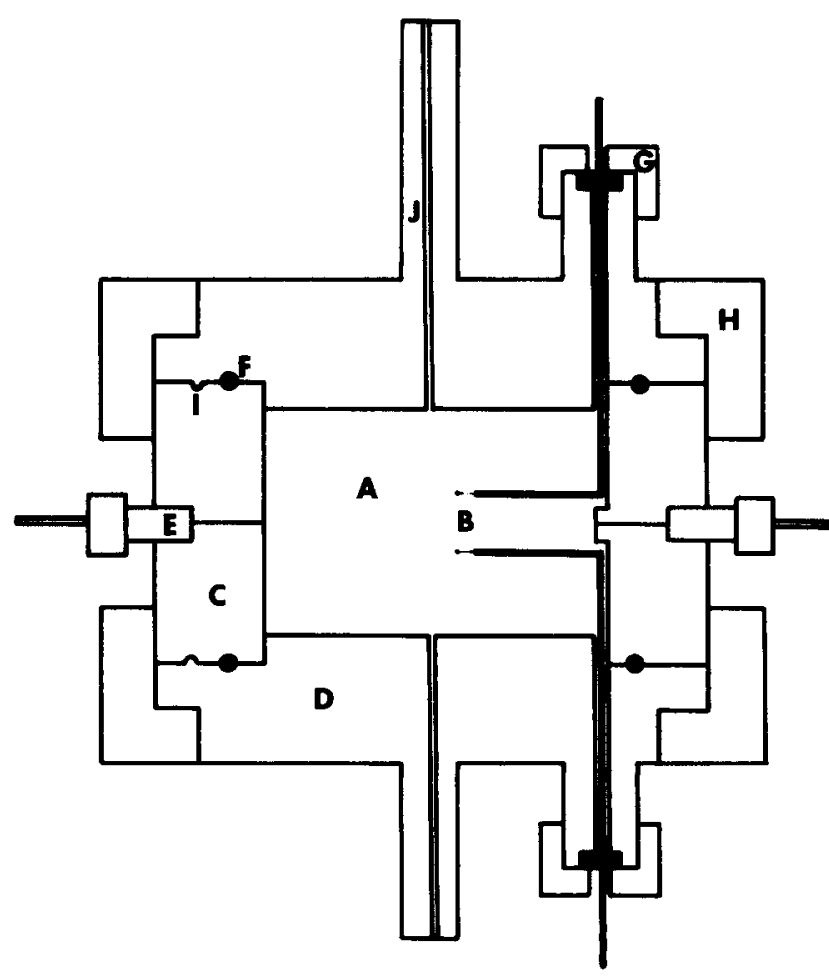

FIG. 1. Schematic diagram of boundary sharpening cell. A. Cell chamber. B. Thermocouples. C. Teflon body. D. Teflon end cap. E. Withdrawal ports. F. O-ring. G. Septum and cap for thermocouple lead seal to the tubes attached to micrometer heads. H. Brass securing ring. I. Alignment pin. J. Filling port. 
the cell was a modification of the boundary sharpening or Tiselius diffusion cell. The cell and experimental technique were the same as those used previously and are adequately described elsewhere. ${ }^{2}$ For clarity of discussion just the concepts of the experiment are repeated here. To measure the heat of transport of a binary mixture of mean mass fraction $\left\langle w_{1}\right\rangle$, an upper-phase mixture of composition $w_{1}^{u}$, and a lower-phase mixture of composition $w_{1}^{l}$, such that $w_{1}^{u}+w_{1}^{l}=\left\langle w_{1}\right\rangle$ and that the density of the upper solution is smaller than that of the lower, are prepared and thermostatted in independent reservoirs within the same constant-temperature air bath as the cell itself. The cylindrical cell, 2.97 $\mathrm{cm}$ inside height and $3.0 \mathrm{~cm}$ i.d., is first completely filled from below with the lower solution. A schematic is shown in Fig. 1. An initial interface is formed between the upper and lower solutions in the bore of a large stopcock and the interface is lowered by withdrawal of fluid through equatorial ports of the cell. As withdrawal of the solution continues, fresh upper solution is introduced in the top of the cell as the rough interface between the upper and lower solutions is lowered in the cell. When the interface approaches the withdrawal plane, fluid from the lower-solution reservoir is allowed to enter at approximately half the total withdrawal rate. The net effect is infusion of fresh upper solution at the top of the cell, fresh lower solution at the bottom of the cell, and withdrawal at cell half-height of the fluid in which diffusion has occurred, thereby creating a sharp horizontal interface. Because the withdrawal rate is faster than diffusion, the interface is stable until the pumps are turned off at the start of the experiment.

There will obviously be temperature nonuniformities introduced into the initially isothermal mixtures by this boundary sharpening technique, but Platt $e t$ al. ${ }^{3}$ have shown that temperature profiles measured in this type of cell, even when quite large initial temperature nonuniformities are present, can be accurately used to obtain heats of transport from the mathematical solution of the diffusion thermoeffect boundary-value problem with isothermal initial conditions by using values measured later than $800 \mathrm{~s}$ into the experiment. This is possible because of the establishment of a steady state between the much more rapid thermal conduction and the slower diffusion thermoeffect or molecular transport heat-transfer modes. Perturbations from this steady state due to initial temperature nonuniformities will always relax back to the appropriate temperature profiles governed by the slower molecular-transport rate.

Temperatures were monitored in the cell using two (one above and one below the initial interface) 40 gage copperConstantan thermocouples of bead diameter less than 0.2 $\mathrm{mm}$. The thermocouples were positioned equidistant from the plane of initial interface formation using micrometers located external to the cell and calibrated to zero for bead positions at the interface. For all the runs reported in this work, the cell location of the temperature probes was $3.0 \pm 0.1 \mathrm{~mm}$ from the interface, corresponding to reduced cell coordinates from bottom to top of $z / a=0.4$ and $z / a=0.6(z$ is vertical coordinate and $a$ is cell height $)$. The micrometer heads were attached to 25 gage stainless-steel sheaths which entered the cell through rubber-Teflon com- posite septum seals, traveled along tiny grooves machined into the wall of the cell, and made right-angle bends to position the measurement beads in the radial center of the cell. The two thermocouples were referenced to each other to measure directly temperature differences. This was done in anticipation of the small values expected and in order to avoid junction errors and problems associated with an icepoint reference. Prior to each run, a thermocouple offset was monitored with the cell filled entirely with the lower-phase solution in order to eliminate spurious readings due to differences and inhomogeneities in the two beads. For 2 to $3 \mathrm{~h}$, the temperature difference between the probes was monitored under isothermal conditions and found to be stable nearly to within the uncertainty of the temperature measurements, 2.6 $\mathrm{mK}$. The value of this isothermal offset was subtracted from all readings during the run.

Thermocouple potential differences were monitored with a Hewlett-Packard, model 3456A, digital multimeter of high input impedance, of $100 \mathrm{nV}$ resolution, and of $\pm 0.008 \%$ of the reading + two counts accuracy. The voltage to temperature conversion was done using a quadratic function originally obtained by fitting the data supplied by the thermocouple-wire manufacturer for the temperaturevoltage response from 0 to $1{ }^{\circ} \mathrm{C}$. Digital output in the form of temperature vs time was then output to a Commodore Super Pet computer where it was logged, saved on disk, and prepared for further analysis. In this manner, data could be logged continuously. The data set used for analysis, however, consisted of points spaced at $100 \mathrm{~s}$ intervals beginning $900 \mathrm{~s}$ into the run and ending after approximately $7200 \mathrm{~s}$.

\section{ANALYSIS PROCEDURE}

As was done by Rowley and Horne, ${ }^{1}$ a numerical procedure was used to solve the partial differential equations describing the diffusion thermoeffect with isothermal initial and vanishing flux boundary conditions. The corresponding composition and temperature equations, simplified ${ }^{1}$ for the cell geometry, types of fluids being studied, and experimental arrangement are

$$
\begin{aligned}
& \rho\left(\frac{\partial w_{1}}{\partial t}\right)=\left\{\frac{\partial\left[\rho D\left(\partial w_{1} / \partial z\right)\right]}{\partial z}\right\}, \\
& \rho C_{p}\left(\frac{\partial T}{\partial t}\right) \\
& =\left\{\frac{\partial[k(\partial T / \partial z)]}{\partial z}\right\}+\left\{\frac{\partial\left[\rho D Q_{1}^{*}\left(\partial w_{1} / \partial z\right)\right]}{\partial z}\right\} \\
& \quad+\left[\rho D M^{3} /\left(M_{1} M_{2}\right)^{2}\right]\left(\frac{\partial^{2} H^{E}}{\partial x_{1}^{2}}\right)\left(\frac{\partial w_{1}}{\partial z}\right)^{2},
\end{aligned}
$$

where $\rho$ is density, $C_{p}$ is specific heat, $k$ is thermal conductivity, $D$ is mutual diffusivity, $M$ is mean molecular weight of the mixture, $M_{i}$ is molecular weight of component $i, x_{1}$ is mole fraction of component 1 , and $H^{E}$ is the molar excess enthalpy. A Crank-Nicholson technique was used as before and the numerical solution was integrated into a nonlinear, weighted, least-squares fitting program which allowed determination of $Q_{1}^{*}$ from the measured temperature differences at fixed and known thermocouple locations as a func- 
TABLE I. Thermophysical properties for binary mixtures composed of toluene (1), chlorobenzene (2), and bromobenzene (3) at $1 \mathrm{~atm}$.

\begin{tabular}{|c|c|c|c|c|c|c|c|c|}
\hline \multicolumn{9}{|c|}{ (A) Density (Ref. 5) $\left(10^{-3} \mathrm{~kg} \mathrm{~m}^{-3}\right)$} \\
\hline \multicolumn{9}{|c|}{$\rho=M /\left(x_{i} V_{i}^{0}+x_{j} V_{j}^{0}+V^{E}\right) ; \quad V^{E}=x_{i} x_{j} \sum_{n=0}^{3} A_{n}\left(1-2 x_{i}\right)^{n}$} \\
\hline \multirow[b]{2}{*}{$i$} & \multirow[b]{2}{*}{$j$} & \multirow[b]{2}{*}{$T / \mathrm{K}$} & \multicolumn{6}{|c|}{ Units $=10^{-3} \mathrm{~m}^{3} \mathrm{~kg}^{-1}$} \\
\hline & & & $V_{i}^{0}$ & $V_{j}^{0}$ & $A_{0}$ & $A_{1}$ & $A_{2}$ & $A_{3}$ \\
\hline 1 & 2 & 298 & 107.26 & 102.05 & -0.172 & -0.01 & 0 & 0 \\
\hline 1 & 2 & 308 & 108.26 & 103.15 & -0.222 & 0 & 0 & 0 \\
\hline 1 & 3 & 308 & 108.26 & 106.91 & -1.052 & 0.28 & -0.08 & 0.02 \\
\hline 2 & 3 & 298 & 90.15 & 105.96 & 16.003 & -0.35 & -0.50 & 0.05 \\
\hline 2 & 3 & 308 & 90.90 & 106.91 & 16.539 & -0.35 & -0.53 & 0.06 \\
\hline
\end{tabular}

(B) Specific heat (Refs. 6 and 7$)\left(\mathrm{kJ} \mathrm{kg}^{-1} \mathrm{~K}^{-1}\right)$

$C_{p}=\left(x_{i} C_{p, i}^{0}+x_{j} C_{p, j}^{0}+C_{p}^{E}\right) / M ; \quad C_{p}^{E}=x_{i} x_{j} \sum_{n=0}^{3} B_{n}\left(1-2 x_{i}\right)^{n}$

\begin{tabular}{rrrrrrrr}
$i$ & $j$ & $T / \mathrm{K}$ & $C_{P, i}^{0}$ & $C_{P, j}^{0}$ & \multicolumn{1}{c}{$B_{0}$} & \multicolumn{1}{c}{$B_{1}$} & \multicolumn{1}{c}{$B_{2}$} \\
\hline 1 & 2 & 298 & 152.39 & 125.12 & 10.987 & -5.44 & 0.57 \\
1 & 2 & 308 & 155.13 & 125.13 & 10.987 & -5.44 & 0.57 \\
1 & 3 & 308 & 155.13 & 152.94 & 7.907 & 6.51 & 12.12 \\
2 & 3 & 298 & 125.12 & 151.53 & -8.186 & 6.71 & 5.90 \\
2 & 3 & 308 & 125.13 & 152.94 & -8.186 & 6.71 & 5.90
\end{tabular}

(C) Molar excess enthalpy (Ref. 7) $\left(\mathrm{J} \mathrm{mol}^{-1}\right)$

$H^{E}=x_{i} x_{j} \sum_{n=0}^{2} C_{n}\left(1-2 x_{i}\right)^{n}$

\begin{tabular}{rrrrrr}
$i$ & $j$ & $T / \mathrm{K}$ & \multicolumn{1}{c}{$C_{0}$} & \multicolumn{1}{c}{$C_{1}$} & \multicolumn{1}{c}{$C_{2}$} \\
\hline 1 & 2 & 298 & -420.76 & 62.88 & -104.51 \\
1 & 2 & 308 & -310.94 & 8.50 & 110.23 \\
1 & 3 & 308 & -255.90 & -33.15 & 81.42 \\
2 & 3 & 298 & 81.21 & -20.03 & 628.95 \\
2 & 3 & 308 & -0.65 & 47.07 & 687.95
\end{tabular}

(D) Mutual diffusivity (Ref. 8) $\left(10^{-9} \mathrm{~m}^{2} \mathrm{~s}^{-1}\right)$

$D=D_{j} w_{i}+D_{i} w_{j}$

\begin{tabular}{lllll}
$i$ & $j$ & $T / \mathrm{K}$ & $D_{i}$ & $D_{j}$ \\
\hline 1 & 2 & 298 & 1.678 & 2.206 \\
1 & 2 & 308 & 1.951 & 2.551 \\
1 & 3 & 308 & 1.533 & 2.454 \\
2 & 3 & 298 & 1.265 & 1.641 \\
2 & 3 & 308 & 1.478 & 1.908
\end{tabular}

(E) Thermal conductivity (Refs. 9-11) $\left(\mathrm{mW} \mathrm{m}^{-1} \mathrm{~K}^{-1}\right)$

\begin{tabular}{cccccccc}
\multicolumn{6}{c}{$k=w_{i} k_{i}^{0}+w_{j} k_{j}^{0}+k^{E} ;$} & \multicolumn{5}{c}{$k^{E}=w_{i} w_{j}\left[G_{j i}\left(k_{i j}-k_{i}^{0}\right) /\left(w_{i}+w_{j} G_{j i}\right)+G_{i j}\left(k_{i j}-k_{j}^{0}\right) /\left(w_{j}+w_{i} G_{i j}\right)\right]$} \\
$i$ & $j$ & $T / \mathbf{K}$ & $k_{i}^{0}$ & $k_{j}^{0}$ & $k_{i j}$ & $G_{i j}$ & $G_{j i}$ \\
\hline 1 & 2 & 298 & 132.68 & 126.37 & 128.66 & 0.9638 & 1.0717 \\
1 & 2 & 308 & 129.92 & 124.10 & 126.22 & 0.9650 & 1.0693 \\
1 & 3 & 308 & 129.92 & 108.83 & 113.82 & 0.9910 & 1.0582 \\
2 & 3 & 298 & 126.37 & 110.81 & 115.74 & 0.9595 & 1.0292 \\
2 & 3 & 308 & 124.10 & 108.83 & 113.67 & 0.9608 & 1.0283 \\
\hline
\end{tabular}

tion of time. Temperatures calculated at the grid points corresponding to the locations of the centers of the thermocouple beads were subtracted from each other and these differences at the time rows corresponding to measured times were used in comparison to measured values to minimize the sum of the squares of the residuals, thereby obtaining bestfitted values of $Q_{1}^{*}$.
Also required in the analysis are the values of the thermophysical properties appearing in Eqs. (1) and (2). Table I contains the property correlations and constants that were used to compute the thermophysical properties at each gridpoint composition in the numerical solution. Only the mean temperature of the run was used in the evaluation of the properties since the induced temperature profiles are small 
TABLE II. Heats of transport measured in binary mixtures of toluene (1), chlorobenzene (2), and bromobenzene (3) at 1 atm.

\begin{tabular}{lcccccc}
\hline \hline$i$ & $j$ & $\left\langle w_{i}\right)$ & $T / \mathrm{K}$ & $-Q_{i}^{*} /\left(\mathrm{kJ} \mathrm{kg}^{-1}\right)$ & $1+\Gamma_{i i}$ & $-\Omega_{0 i} /\left(10^{-7} \mathrm{~kg} \mathrm{~m}^{-1} \mathrm{~s}^{-1}\right)$ \\
\hline 1 & 2 & 0.3000 & 298 & 6.21 & 1.020 & 1.07 \\
1 & 2 & 0.3996 & 298 & 12.08 & 1.020 & 2.29 \\
1 & 2 & 0.5000 & 298 & 10.50 & 1.026 & 1.99 \\
1 & 2 & 0.6025 & 298 & 14.98 & 1.025 & 2.63 \\
1 & 2 & 0.3000 & 308 & 16.19 & 1.020 & 3.07 \\
1 & 2 & 0.3000 & 308 & 15.38 & 1.020 & 2.92 \\
1 & 2 & 0.4000 & 308 & 16.59 & 1.024 & 3.45 \\
1 & 2 & 0.5500 & 308 & 20.81 & 1.026 & 4.22 \\
1 & 2 & 0.6000 & 308 & 24.82 & 1.025 & 4.80 \\
1 & 3 & 0.2010 & 308 & 23.35 & 1.019 & 3.90 \\
1 & 3 & 0.7000 & 308 & 19.33 & 1.018 & 3.30 \\
2 & 3 & 0.2000 & 298 & 14.20 & 1.0 & 2.34 \\
2 & 3 & 0.3000 & 298 & 9.41 & 1.0 & 2.07 \\
2 & 3 & 0.5000 & 298 & 8.55 & 1.0 & 2.14 \\
2 & 3 & 0.6000 & 298 & 9.31 & 1.0 & 2.24 \\
2 & 3 & 0.2000 & 308 & 23.71 & 1.0 & 4.20 \\
2 & 3 & 0.3000 & 308 & 24.84 & 1.0 & 5.74 \\
2 & 3 & 0.5000 & 308 & 12.30 & 1.0 & 3.37 \\
2 & 3 & 0.6000 & 308 & 15.08 & 1.0 & 3.98 \\
2 & 3 & 0.7000 & 308 & 16.57 & 1.0 & 3.84 \\
\hline \hline
\end{tabular}

(less than $\pm 0.3 \mathrm{~K}$ deviation from the initial value) and lead to insignificant effects in property values. This also serves to decouple Eq. (1) from Eq. (2), allowing solution of the composition equation at each time row independent of the temperature equation solution. Because the relaxation time of diffusion is slow compared to that of thermal conduction, the composition profile of the previous time step could be used in the temperature solution without loss of accuracy. Initially, very short time steps $(2 \mathrm{~s})$ were used when the composition gradient was steep. These were gradually expanded to $100 \mathrm{~s}$ steps as the composition gradient was diminished.

TABLE III. Thermal-diffusion coefficients and ratios obtained from Onsager reciprocity at $1 \mathrm{~atm}$.

\begin{tabular}{llllll}
\hline \hline$i$ & $j$ & $\left\langle w_{i}\right\rangle$ & $T / \mathrm{K}$ & $-D_{T, i} /\left(10^{-10} \mathrm{~m}^{2} \mathrm{~s}^{-1}\right)$ & $-K_{T, i}$ \\
\hline 1 & 2 & 0.3000 & 298 & 1.05 & 0.0565 \\
1 & 2 & 0.3996 & 298 & 2.31 & 0.120 \\
1 & 2 & 0.5000 & 298 & 2.06 & 0.104 \\
1 & 2 & 0.6025 & 298 & 2.79 & 0.138 \\
1 & 2 & 0.3000 & 308 & 3.05 & 0.141 \\
1 & 2 & 0.3000 & 308 & 2.90 & 0.134 \\
1 & 2 & 0.4000 & 308 & 3.52 & 0.158 \\
1 & 2 & 0.5500 & 308 & 4.47 & 0.193 \\
1 & 2 & 0.6000 & 308 & 5.14 & 0.220 \\
1 & 3 & 0.2010 & 308 & 3.04 & 0.167 \\
1 & 3 & 0.7000 & 308 & 3.38 & 0.149 \\
2 & 3 & 0.2000 & 298 & 1.68 & 0.124 \\
2 & 3 & 0.3000 & 298 & 1.48 & 0.109 \\
2 & 3 & 0.5000 & 298 & 1.65 & 0.114 \\
2 & 3 & 0.6000 & 298 & 1.74 & 0.120 \\
2 & 3 & 0.2000 & 308 & 3.05 & 0.192 \\
2 & 3 & 0.3000 & 308 & 4.29 & 0.263 \\
2 & 3 & 0.5000 & 308 & 2.62 & 0.155 \\
2 & 3 & 0.6000 & 308 & 3.12 & 0.185 \\
2 & 3 & 0.7000 & 308 & 3.04 & 0.180 \\
\hline \hline
\end{tabular}

\section{RESULTS}

The heats of transport obtained are shown in Table II. Also shown are the values of the heat-mass Onsager coefficient $\Omega_{0 i}$ obtained from the identity

$$
\Omega_{0 i}=\rho D Q_{i}^{*} M_{i} M_{j} w_{i} w_{j} /\left[M R T\left(1+\Gamma_{i i}\right)\right],
$$

where the values of $\Gamma_{i i}$ for the toluene + chlorobenzene and toluene + bromobenzene binaries were calculated from

$$
\begin{aligned}
& \Gamma_{11}=x_{1} x_{2}\left(0.12265-0.0213 x_{2}-0.0424 x_{2}^{2}\right), \\
& \Gamma_{11}=x_{1} x_{3}\left(0.5372-0.1285 x_{3}-0.2204 x_{3}^{2}\right),
\end{aligned}
$$

respectively. Here, subscript $i$ refers to the lowest component number in binaries of toluene (1), chlorobenzene (2), and bromobenzene (3) and $j$ refers to the second component. Also, $\Gamma_{i i}=\left(\partial \ln \gamma_{i} / \partial \ln x_{i}\right)_{T, P}$, where $\gamma_{i}$ is the activity coefficient of component $i$ based on the pure-component standard state. Equations (4) and (5) were fitted using the free energy data of Khosla et al. ${ }^{12}$ As these two binaries are nearly ideal in the thermodynamic sense $\left(\Gamma_{i i}=0\right)$ and the chlorobenzene + bromobenzene binary is expected to be the most ideal of the three, $\Gamma_{22}$ was assumed to be zero in chlorobenzene-bromobenzene mixtures for lack of data. It should be mentioned that the negative sign for all of the heats of transport and Onsager Coefficients is an artifact of the way we have numbered the components since it is easily shown that $Q_{2}^{*}=-Q_{1}^{*}$ in a binary mixture of components 1 and 2 . The negative sign in conjunction with the numbering system used here indicates that heat is transported down the composition gradient of the more-dense component or up the gradient of the less-dense component.

Based on Onsager reciprocity $\Omega_{i 0}=\Omega_{0 i}$, which has been proven experimentally under similar circumstances, ${ }^{13}$ one can calculate thermal diffusion coefficients $D_{T}$ and thermal diffusion ratios $K_{T}$ from diffusion thermoeffect measurements. The relationships between these latter coefficients and the Onsager coefficient are 


$$
\begin{aligned}
D_{T, i} & =\Omega_{i 0} / \rho, \\
K_{T, i} & =D_{T, i} / D=\Omega_{i 0} / \rho D .
\end{aligned}
$$

The values obtained are shown in Table III.

\section{DISCUSSION OF RESULTS}

As Platt et al. have shown, there are two independent heats of transport in a ternary mixture. If the heat $q$ and mass (relative to the center of mass) $\mathbf{j}_{i}$ flux equations are written in terms of Onsager coefficients for an $n$-component system

$$
\begin{aligned}
-\mathbf{q} & =\Omega_{00} \nabla \ln T+\sum_{j=1}^{n-1} \sum_{k=1}^{n-1} \sum_{l=1}^{n-1} \Omega_{0 j} f_{j k} \mu_{k l} \nabla w_{l}, \\
-\mathbf{j}_{i} & =\Omega_{i 0} \nabla \ln T \\
& +\sum_{j=1}^{n-1} \sum_{k=1}^{n-1} \sum_{l=1}^{n-1} \Omega_{i j} f_{j k} \mu_{k l} \nabla w_{l} \quad(i=1,2, \ldots, n-1),
\end{aligned}
$$

where $f_{j k}$ and $\mu_{k l}$ are given by

$$
\begin{aligned}
& f_{j k}=\delta_{j k}+w_{k} / w_{n}, \\
& \mu_{k l}=\left(\frac{\partial \mu_{k}}{\partial w_{l}}\right)_{T, P},
\end{aligned}
$$

and $\delta_{j k}$ is the Kronecker delta, then heats of transport can be written in terms of the Onsager coefficients as

$$
\begin{aligned}
& Q_{1}^{*}=\left(\Omega_{01} \Omega_{22}-\Omega_{02} \Omega_{21}\right) /\left(\Omega_{11} \Omega_{22}-\Omega_{12} \Omega_{21}\right), \\
& Q_{2}^{*}=\left(\Omega_{02} \Omega_{11}-\Omega_{01} \Omega_{12}\right) /\left(\Omega_{11} \Omega_{22}-\Omega_{12} \Omega_{21}\right) .
\end{aligned}
$$

These equations may be inverted and written as

$$
\begin{aligned}
& \Omega_{01}=\Omega_{11} Q_{1}^{*}+\Omega_{21} Q_{2}^{*}, \\
& \Omega_{02}=\Omega_{12} Q_{1}^{*}+\Omega_{22} Q_{2}^{*} .
\end{aligned}
$$

On the other hand, the binary heat of transport is a simple ratio of two Onsager coefficients

$$
Q_{1}^{*}=\Omega_{01} / \Omega_{11} \text {. }
$$

The Onsager coefficients themselves are a function of composition, as can be seen from the results of Table II, so there is no obvious way to correlate Onsager coefficients in ternary mixtures with those in the constituent binaries. To make matters conceptually simpler, we attempt to correlate thermal diffusion ratios rather than Onsager coefficients, though the two are related for a binary as in Eq. (7) and for a ternary system by ${ }^{4}$

$$
K_{T, i}=D_{T, i} / D_{i i} \quad(i=1,2) .
$$

The relationships used here between transport and Onsager coefficients are the same as those used by Platt $e t a l^{2}$ so that the multicomponent flux equations, Eqs. (8) and (9), can be rewritten in terms of transport coefficients as

$$
-\mathbf{q}=k \nabla T+\sum_{k=1}^{n-1} \sum_{l=1}^{n-1} \rho Q_{k}^{*} D_{k l} \nabla w_{l},
$$

$-\mathbf{j}_{i}=\rho D_{T, i} \nabla \ln T+\sum_{l=1}^{n-1} \rho D_{i l} \nabla w_{l} \quad(i=1,2, \ldots, n-1)$,

where obviously

$$
k=\Omega_{00} / T
$$

and

$$
\rho D_{i l}=\sum_{j=1}^{n-1} \sum_{k=1}^{n-1} \Omega_{i j} f_{j k} \mu_{k l} .
$$

In a binary mixture one could speak of the thermaldiffusion ratio of either component in the mixture since $\mathbf{j}_{1}+\mathbf{j}_{2}=0$ requires that

$$
K_{T, 1}=-K_{T, 2} \text {. }
$$

In an attempt to correlate ternary thermal-diffusion ratios in terms of binary values, all the binary data were fitted to the form

$$
K_{T, i}=w_{j} K_{T, i}^{\infty}+w_{i} K_{T, i}^{0}+w_{i} w_{j} \alpha_{i j},
$$

where again $i$ refers to the lowest component number in binaries of toluene (1), chlorobenzene (2), and bromobenzene (3) and $j$ refers to the other component. In this manner, $K_{T, i}^{0}$ can be thought of as the value in the pure-component- $i$ limit and $K_{T, i}^{\infty}$ can be thought of as the infinitely dilute value, which according to Eq. (18) must be equal to $-K_{T, j}^{0}$ in the $i-j$ binary. Thus, it is required that $K_{T, i}^{0}$ be the same regardless of which of the two possible binaries containing component $i$ it was determined from. Similarly, we require that $K_{T, 2}^{0}$ be the same in both possible binaries containing component 2 . And, $K_{T, 3}^{0}$ is a pure-3 limit equal to $-K_{T, 2}^{\infty}$ in the $2-3$ binary and $-K_{T, 1}^{\infty}$ in the $1-3$ binary. Thus, we fit six parameters to describe all three binaries: three "pure component" thermal diffusion ratios and three mixture excess related constants, $\alpha_{i j}$, one for each binary.

While better fits of the binary data could certainly have been achieved by a nonsymmetric functionality for the excess portion, too many adjustable parameters would make fitting of physically reasonable pure-component values suspect. Even so, without additional information much closer to the pure component limits, we do not place much confidence in the physical significance of the $K_{T, i}^{0}$ values and only use this procedure in an attempt to develop a rough estimation procedure for the ternary thermal diffusion ratios from the single independent values measured in binary experiments. The true behavior of these cross-transport coefficients in the pure component limit, however, is an interesting and yet unstudied problem that deserves further attention.

A simultaneous fit of Eq. (23) to the $K_{T, i}$ values measured in the three binary mixtures toluene $(1)+$ chlorobenzene ( 2 ), toluene (1) + bromobenzene ( 2 ), and chlorobenzene (2) + bromobenzene (3) yielded the values $K_{T, 1}^{0}=0.1596, \quad K_{T, 2}^{0}=0.0523, \quad K_{T, 3}^{0}=-0.1905$, $\alpha_{12}=0.5659, \alpha_{13}=-0.0995$, and $\alpha_{23}=0.3152$. These values were then used to estimate ternary values for $K_{T, 1}$ and $K_{T, 2}$ at the same compositions reported by Platt et al. ${ }^{2}$ To estimate the values in the ternary mixtures, we used

$$
\begin{aligned}
K_{T, 1}= & w_{1} K_{T, 1}^{0}-w_{2} K_{T, 2}^{0}-w_{3} K_{T, 3}^{0} \\
& +\alpha_{12} w_{1} w_{2}+\alpha_{13} w_{1} w_{3}, \\
K_{T, 2}= & w_{2} K_{T, 2}^{0}-w_{1} K_{T, 1}^{0}-w_{3} K_{T, 3}^{0} \\
& +\alpha_{23} w_{2} w_{3}-\alpha_{12} w_{1} w_{2},
\end{aligned}
$$

where the minus signs arise from analogs of Eq. (22) for each binary. Thus, the three corners of a $K_{T, 1}$ vs ternary composition diagram are defined by $K_{T, 1}^{0}$ and $K_{T, 1}^{\infty}$ in the 12 binary (which is equal to $-K_{T, 2}^{0}$ ), and $K_{T, 1}^{\infty}$ in the 1-3 
TABLE IV. Thermal-diffusion ratios correlated for binary mixtures and used to estimate values in ternary mixtures ${ }^{2}$ of toluene (1), chlorobenzene (2), and bromobenzene (3) at $35^{\circ} \mathrm{C}$ and $1 \mathrm{~atm}$.

\begin{tabular}{lccccc}
\hline \hline$\left(w_{1}\right\rangle$ & $\left\langle w_{2}\right\rangle$ & $-K_{T, 1}$ (expt) & $-K_{T, 1}$ (calc) & $-K_{T, 2}$ (expt) $-K_{T, 2}$ (calc) \\
\hline 0.300 & 0.700 & 0.141 & 0.130 & $\ldots$ & $\ldots$ \\
0.300 & 0.700 & 0.134 & 0.130 & $\ldots$ & $\ldots$ \\
0.400 & 0.600 & 0.158 & 0.168 & $\ldots$ & $\ldots$ \\
0.550 & 0.450 & 0.193 & 0.204 & $\ldots$ & $\ldots$ \\
0.600 & 0.400 & 0.220 & 0.211 & $\ldots$ & $\ldots$ \\
0.201 & 0.799 & 0.167 & 0.168 & $\ldots$ & $\ldots$ \\
0.700 & 0.300 & 0.149 & 0.148 & $\ldots$ & $\ldots$ \\
0 & 0.200 & $\ldots$ & $\ldots$ & 0.192 & 0.213 \\
0 & 0.300 & $\ldots$ & $\ldots$ & 0.263 & 0.215 \\
0 & 0.500 & $\ldots$ & $\ldots$ & 0.155 & 0.200 \\
0 & 0.600 & $\ldots$ & $\ldots$ & 0.185 & 0.183 \\
0 & 0.700 & $\ldots$ & $\ldots$ & 0.180 & 0.160 \\
0.200 & 0.200 & 0.145 & 0.146 & -0.040 & 0.108 \\
0.200 & 0.400 & 0.095 & 0.125 & 0.048 & 0.070 \\
0.200 & 0.600 & 0.074 & 0.103 & 0.049 & 0.075 \\
0.325 & 0.175 & 0.128 & 0.154 & 0.024 & 0.048 \\
0.326 & 0.274 & 0.141 & 0.151 & 0.055 & 0.022 \\
0.400 & 0.100 & 0.194 & 0.157 & 0.029 & 0.030 \\
0.400 & 0.300 & 0.156 & 0.161 & 0.091 & -0.030 \\
0.450 & 0.450 & 0.175 & 0.177 & -0.055 & -0.130 \\
0.600 & 0.250 & 0.177 & 0.187 & 0.001 & -0.127 \\
\hline \hline
\end{tabular}

binary (which is equal to $-K_{T, 3}^{0}$ ).

Table IV shows both the results of the correlation of the binary $K_{T}$ values with Eq. (23) and the prediction of ternary values from Eqs. (24) and (25). The predicted values are compared to values determined from the measured heats of transport reported by Platt et al. ${ }^{2}$ Agreement seems adequate for a rough estimation technique of this sort. It should also be pointed out that although we expect the accuracy of the binary measurements reported here to be $3 \%-4 \%$, the accuracy of the ternary experiments are considerably less since $Q_{1}^{*}$ and $Q_{2}^{*}$ must be simultaneously fit. Furthermore, in the conversion from heats of transport to Onsager coefficients and then finally to thermal diffusion ratios, errors associated with the determination of $\Gamma_{i i}$ and the errors in the diffusivity and density due to inadequacies of the correlations in Table I, introduce considerable more uncertainty. We, therefore, do not expect the binary $K_{T}$ values to be any more accurate than about $10 \%$ and the ternary values than about $15 \%$. The ternary estimation method of Eqs. (24) and (25) therefore appear adequate.

\section{CONCLUSIONS}

Measurements of the diffusion thermoeffect in binary liquid mixtures of toluene, chlorobenzene, and bromobenzene have been made at 298 and $308 \mathrm{~K}$ at $1 \mathrm{~atm}$. Reduction of temperature response data vs time yields the heat of transport which was found to be typically on the order of 10-20 $\mathrm{kJ} / \mathrm{kg}$. While the diffusion-induced temperature response is small due to back thermal conduction, heats of transport are substantial in value and indicate considerable heat transfer by the molecular mode.

Onsager coefficients and thermal-diffusion coefficients and ratios have been calculated from the heats of transport. Study of these three binary mixtures allows for the first time investigation of the relationship between the single, independent thermal-diffusion ratio in binary mixtures and the two, independent, thermal diffusion ratios in ternary mixtures. The binary data can be correlated in terms of pure component limiting thermal-diffusion ratios, which can in turn be used to calculate ternary values. Comparison of ternary values estimated entirely from the binary data with those measured by Platt $e t$ al. ${ }^{2}$ yields reasonable agreement.

\section{ACKNOWLEDGMENT}

Support of this project under National Science Foundation Grant No. CPE-8410937 is gratefully acknowledged.

${ }^{1}$ R. L. Rowley and F. H. Horne, J. Chem. Phys. 72, 131 (1980).

${ }^{2}$ G. Platt, T. Vongvanich, and R. L. Rowley, J. Chem. Phys. 77, 2121 (1982).

${ }^{3}$ G. Platt, T. Vongvanich, and R. L. Rowley, J. Chem. Phys. 77, 2113 (1982).

${ }^{4}$ G. E. Platt, T. Vongvanich, and R. L. Rowley, J. NonEquilib. Thermodyn. 8, 1 (1983).

${ }^{5}$ R. K. Nigam and P. P. Singh, Trans. Faraday Soc. 65, 950 (1969).

${ }^{6} \mathrm{~J}$. W. Williams and F. Daniels, J. Am. Chem. Soc. 47, 1490 (1925).

'J. J. Christensen, R. W. Hanks, and R. M. Izatt, Handbook of Heats of Mixing (Wiley, New York, 1982).

${ }^{8} \mathrm{~J}$. K. Burchard and H. L. Toor, J. Phys. Chem. 66, 2015 (1962).

${ }^{9} \mathrm{D}$. T. Jamieson, J. B. Irving, and J. S. Tudhope, Liquid Thermal Conductivity: A Data Survey to 1973 (HMO, Edinburgh, 1975).

${ }^{10}$ R. L. Rowley, Chem. Eng. Sci. 37, 897 (1982).

${ }^{11} \mathrm{~J}$. Gmehling and U. Onken, Vapor-Liquid-Equilibrium Data Collection; Dachema Chem. Data Series, Vol.1 (Verlag \& Druckerel Friedrich Bischoff, Frankfurt, 1977).

${ }^{12}$ M. P. Khosla, B. S. Mahl, S. L. Chopra, and P. P. Singh, Z. Phys. Chem. (Leipzig) 251, 129 (1972).

${ }^{13}$ R. L. Rowley and F. H. Horne, J. Chem. Phys. 68, 325 (1978). 